\title{
System Description: An Orienteering Strategy to Browse Semantically-Enhanced Educational Wiki Pages
}

\author{
Luciano T.E. Pansanato ${ }^{1}$ and Renata P.M. Fortes ${ }^{2}$ \\ ${ }^{1}$ Universidade Tecnológica Federal do Paraná - Campus de Cornélio Procópio, \\ Av. Alberto Carazzai, 1640, 86300-000 Cornélio Procópio, PR, Brazil \\ ${ }^{2}$ Departamento de Ciências de Computação, Instituto de Ciências Matemáticas e de \\ Computação, Universidade de São Paulo - Campus de São Carlos \\ Caixa Postal 668, 13560-970 São Carlos, SP, Brazil \\ luciano@utfpr.edu.br, renata@icmc.usp.br
}

\begin{abstract}
Wikis have been adopted along the years, aiming to provide an easy and simple support to people keep the information systems on the Internet upto-date, and making possible efficient collaborative authoring. As the number of pages and corresponding contents increases, wiki users face difficulties when browsing for wiki pages. This paper presents a system prototype based on orienteering to browse semantically-enhanced educational wiki pages. The results of a user-based evaluation of the system prototype are also presented.
\end{abstract}

Keywords: Semantic Web, Metadata, Wiki, Interactive Retrieval.

\section{Introduction}

Information seeking activities play an important role in a wide range of tasks based on information systems. In Wikis, the support for information seeking activities is important to enable users to find the information they need. However, browsing for information in Wikis may be difficult when users are supported only by the traditional keyword search paradigm.

The keyword search paradigm is popular because of its ability to identify quickly the pages containing specific information. However, keyword search is not an efficient means of finding information in several situations. For example: (a) a user may be uncertain of what he is looking for until the available options are presented; (b) the target cannot be expressed by keywords or (c) the exact terminology used in the pages is unknown, and (d) in cases where a great deal of information and context should be obtained along with the pages (and not only the final page). In such situations, other paradigms such as traditional browsing may be more useful than keyword search [1].

We have observed this problem with the CoTeia ${ }^{1}[2]$, a Computer Supported Collaborative Learning (CSCL) tool used to complement face-to-face lectures with

\footnotetext{
${ }^{1}$ http://incubadora.fapesp.br/projects/coteia/
} 
collaborative learning activities at Institute of Mathematical Sciences and Computing / University of São Paulo (ICMC/USP). CoTeia is a wiki-based asynchronous collaborative tool analogous to CoWeb [3]. CoTeia users, in particular faculty, frequently look for wiki pages to reuse their material. CoTeia has been used since 2001 and contains a significant amount of teaching material. Although CoTeia provides a keyword search tool, we have observed many cases where users engage in an exhaustive browsing to find what they are seeking.

We agree that typical wiki-based environments, such as CoTeia, can be enhanced by metadata, which may strongly influence information seeking techniques and tools. Semantic MediaWiki is also a well-known wiki behind Semantic Wikipedia [4]. However, the ease in using metadata to find wiki pages (or other kind of information objects) is related to the existence of appropriate support to information seeking strategies. The main objective of our research is to investigate and develop an interactive support inspired by an orienteering strategy [1, 5] to browsing for wiki pages, which uses an infrastructure of Semantic Web. Orienteering denotes a strategy in which people satisfy a particular information need through a sequence of small steps (or actions) to narrow the focus of the goal. At each step, prior information and local context are used to decide the next step.

We have developed a prototype for integrating a variety of tools to support an orienteering strategy. We argue that an information seeking environment should make available several categories of tools (including keyword search), enabling users to choose the appropriate tool or the best combination of tools (that is, the best strategy) in agreement with different levels of users' ability, background, preferences, and kind of information that they are looking for at moment. It enables users to prioritize different ways their choices of tools to each step in an orienteering strategy. We expected that users take advantage of this kind of environment to carry out information seeking tasks as an alternative to the one-size-fits-all approach of the keyword search paradigm.

Section 2 describes the orienteering strategy and the techniques we have explored to support information seeking activities. Sections 3 and 4 present the prototype and a preliminary user-based evaluation which points to its effectiveness. Sections 5 and 6 are dedicated to related work and conclusions respectively.

\section{Strategies and Tools}

In our work, we exploit the levels of information seeking activities as described by Bates [6]. Based on empirical studies of the information seeking behavior of experienced library users, Bates distinguishes four levels of activity:

1. A move is an identifiable thought or action that is a part of information seeking. For example, locating some portion of text in a wiki page.

2. A tactic is one or a handful of moves made to further an information seeking activity. For example, broadening or narrowing a query to retrieve a larger or smaller number of wiki pages.

3. A stratagem is a complex set of moves and/or tactics, and generally involves both a particular information domain anticipated to be productive by the user, and a 
mode of tackling the particular organization of that domain. For example, finding all wiki pages which are related to a particular course.

4. A strategy is a plan for satisfying an information need, and may include combinations of all the previously mentioned types of information seeking activities. For example, performing a keyword search to retrieve wiki pages related to specific terms describing a subject, browsing through those considered relevant and then finding references in the text to books and articles.

We are interested in providing an interactive support to help users to find wiki pages at CoTeia. The support provided for moves and tactics is implemented as a set of tools and these are integrated in an environment. Combining the tools users may compose stratagems and strategies. Thus, in this work a tool is a software program that implements one or more methods or techniques to support an information seeking activity; and an environment is a software program that integrates diverse tools.

Subsection 2.1 describes the underlying orienteering strategy used to integrate the tools which has influenced the choice of the tools as well as some extensions. The tools are briefly discussed in Subsection 2.2.

\subsection{Orienteering Strategy}

Orienteering is a sport of finding one's way across country on foot using a map and a compass. The main strategy consists in using the information on the current position to make better choices about the way to reach the next checkpoint or the final target. Furthermore, the orienteer must constantly concentrate, make decisions, and keep track of the path covered.

A similar strategy has been described in studies of information seeking behavior in the literature $[1,5]$. Orienteering involves using both prior and contextual information to narrow in on the actual information target, often in a series of steps (moves/tactics/stratagems, according to Bates, or simply actions), without specifying the entire information need up front. For example, first one can submit a query to a search engine to get into the proximity of the information that satisfy the target information need, and then explore the links retrieved to find the desired information. Other works also described this strategy [7, 8], though not under that name.

We have elected the following categories of techniques that could help the user to engage in an orienteering strategy: combining browsing and searching; showing context; previewing content; keeping interaction history; and narrowing toward the goal. The latter is a key feature of an orienteering strategy. It consists in starting with a general query and using small steps to narrow down the information space until the user finds what they are looking for.

\subsection{Orienteering Tools}

We have implemented common tools and extended others to incorporate metadata in their mechanism, i.e. a Resource Description Framework (RDF) model about a collection of wiki pages used in educational activities. Methods for automatic 
metadata generation were explored to populate the RDF model as described in previous work $[9,10]$. The following tools were implemented:

- Keyword search. This tool is a search engine which searches previously indexed wiki pages for specified keywords and returns a list of those where the keywords were found. In spite of this approach being straightforward, it works for its purpose: to obtain a list of wiki pages relating to the keywords the user entered. Some search engines can use relevance ranking that many users find disconcerting: some pages can be ranked high even if they do not contain all the keywords.

- Facet browse. Faceted browsing is one way to use faceted metadata to allow users to find information. Metadata can have several facets: attributes in various orthogonal sets of categories. For example, in the domain of educational wiki pages, possible facets might be authors (professors or students). This tool (Facet browse) allows users to filter a set of items (e.g. wiki pages) by progressively selecting from only valid values (instances) of facets. The list of valid values is filtered to show only those that have results available. Thus, it is impossible to get an empty result. The combination of facets and hierarchy can help the user to decide how to start and to explore the collection.

- Highlight. This tool displays the occurrences of query terms within the context of the document retrieved. It is useful to support local exploration because most users do not read pages carefully when they scan text for what they are looking for. We extend this technique to highlight also names, locations, email addresses, and phone numbers. The knowledge about people's names and locations is harvested from ICMC/USP website and added inside the RDF model.

- Flag. This tool implements a preview technique that consists in automatically flagging a result which contains certain content so it can be found easily among other results. For example, results of documents that contain names, locations, email addresses, dates, or times. It is useful for users to be able to see at a glance whether the results they get in response to an action have a particular content.

- Work memory. The underlying technique of this tool consists in providing a special memory resource which can store results and some operations to handle them. Basically, we implemented the same memory functions of a typical calculator: M+ (sum), M- (difference), MR (recall), and MC (clear). This support is important in cases where the goal consists of a collection of results that should be obtained from a variety of different strategies, not just at the end of a strategy. For example, collecting links to wiki pages which contains educational material (for reuse) may involve the storage of intermediary results to compose the final list of those considered relevant.

- Sort. This tool sorts the results by relevance, as determined by the number and location of matched words in the wiki page, and by any metadata of wiki pages in the RDF model. While all search engines sort results by relevance as default, a few of them can sort by other option (e.g. Ask, http://www.ask.com/, allows to sort saved results by date or title).

- Group. Some search engines attempt to classify results automatically into concepts (or domains), such as Vivísimo, http://vivisimo.com/. The drawback of this 
technique is that the categories automatically generated are not always well organized. This tool (Group) implements a straightforward improvement by exploiting the available metadata in the RDF model to organize the results according to the same category layout that is used by the tool for faceted browsing.

- Undo and Restart. The reverse of actions is supported by means of undoing and starting over the task. The Undo tool allows user to cancel any performed action and consequently, the results will reflect on the new sequence. The Restart tool allows user to begin again from scratch. These tools help to keep the interaction process under user control.

\section{Prototype}

We have developed a prototype which integrates the orienteering tools described in previous section. The prototype has been used to browse approximately 4,230 wiki pages stored in two CoTeia repositories ${ }^{2}$. Fig. 1 shows an example of interaction with the prototype. The interface is divided into three parts: the set of tools on the left, the history of actions on the top right, and the results and content area on the bottom right.

Using a tool often corresponds to a move, tactic, or stratagem (discussed in Section 2), and this interaction is called action in the interface. The sequence of performed actions during a strategy is showed in the history in the top right. The history serves as important aid to reduce working memory load. Any particular information seeking activity may include other behaviors and cognitions that cannot be captured by the prototype.

When the user performs an action, the results (i.e. a list of links to wiki pages in this case) to that action are presented. The next action has effect on the current set of results. This is an important aspect in the interaction process of the user with the prototype: the tools are integrated so that the results obtained in one are used as the input to the next.

Fig. 1 shows the result of a hypothetical interaction in which the user has chosen different tools to look for wiki pages with material (slides) used in a particular course. First, the user selected the term "SCE0225 Hipermídia" (A) in the facet Course to refine the entire collection of all wiki pages to a set of those related with the course. Second, the user performed a keyword search (B) to narrow the current set of wiki pages (from previous tool) to those which contain the word "aula" (lecture). Next, the user sorts the results alphabetically by Title (C) and follows a link to the wiki page entitled "Aula a Aula" (D). The wiki page is showed in the content area (E). Next, the highlight tool is used to highlight the word "Aula" $(F)$ in the content of the wiki page (the highlight tool does not appear in the figure). Finally, the flag tool is used to puts a mark (a red lozenge) $(\mathrm{G})$ on the results which contain one or more people's names.

The prototype was built using PHP and MySQL. We have used AJAX (Asynchronous JavaScript and XML) programming techniques to allow better user interactions in the interface. The RDF model is manipulated and searched using RAP (RDF API for PHP, http://sourceforge.net/projects/rdfapi-php/).

\footnotetext{
2 (1) http://coteia.icmc.usp.br/coteia/ and (2) http://safedevel.icmc.usp.br/coweb/
} 


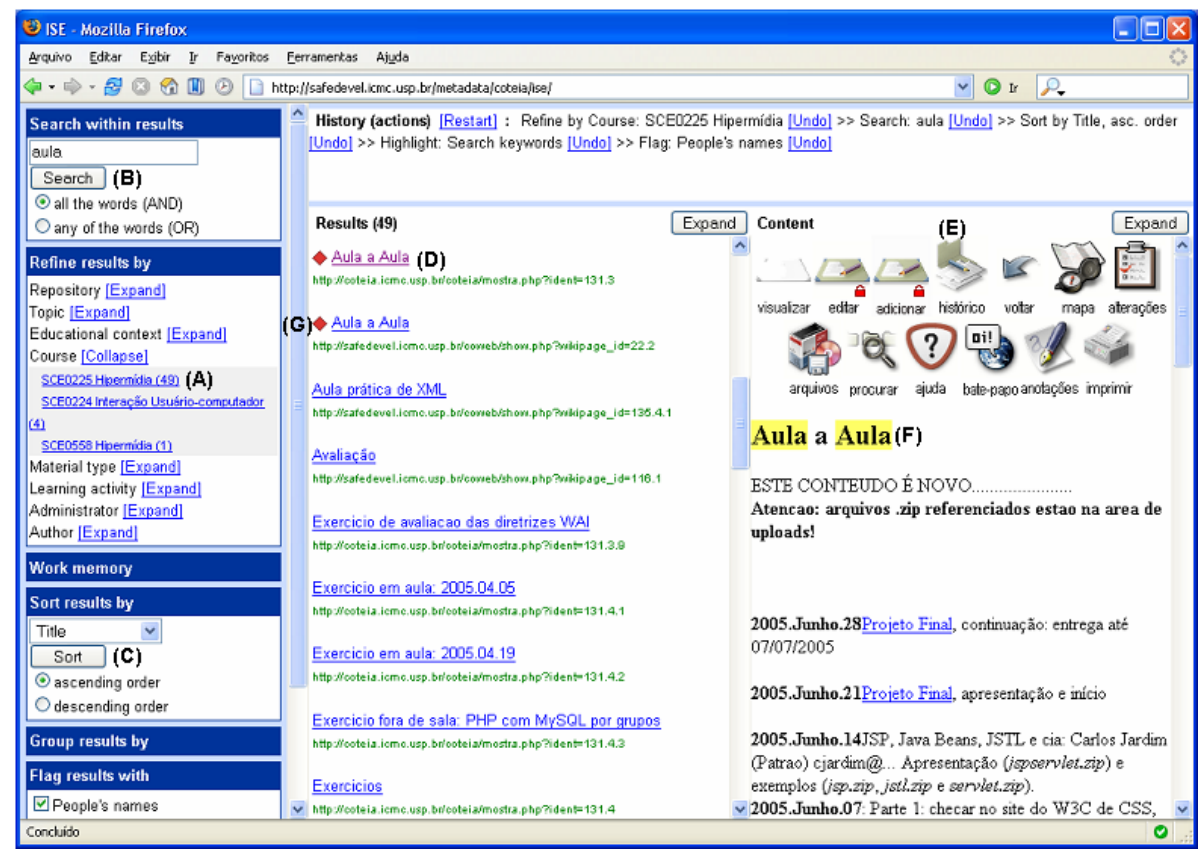

Fig. 1. Screenshot of the prototype

\section{User-Based Evaluation}

This section presents the evaluation we conduct to test and evaluate the prototype, and, through the users' interaction with the prototype, to gain knowledge about it.

\subsection{Aims}

We had three goals for the evaluation. First, we were interested in validating if the users could get relevant information using the prototype. Second, we wanted to confirm if the users use different strategies. Finally, we were interested in studying step-by-step the strategies that users follow to browsing for wiki pages using the prototype and their preferences concerning the available tools.

\subsection{Methodology}

We have combined a variety of methods to gather data about the user experience with the prototype. Our methodology combined: (a) Questionnaire, (b) Think Aloud, (c) Interview, and (d) User Log Recording. The participants consisted of 15 professors (11 women, 4 men) at ICMC/USP. They were all experienced users of search engines, searching for information daily. The participants were regular users of the CoTeia with 2-6 years of experience and weekly they access wiki pages for reading and/or editing. Thus, the participants are potential end-users of the prototype. 
The participants completed a set of tasks that involved browsing for educational material for reuse. We defined five tasks in four different task scenarios (types), based on Shneiderman's definition [11]: specific fact-finding, extended fact-finding, openended browsing and exploration of availability (see Table 1). We were interested in covering the largest number of scenarios using the smallest number of tasks.

The tasks were presented to the participants in agreement with the concept of simulated work task situation [12]. A simulated work task situation is an open description of the context/scenario of a given situation. It then works as the trigger of the participant's information need and the base for relevance judgment. The objective is to ensure the largest possible realism by the involvement of potential users who, based on the simulated work task situation, develop individual and subjective information need interpretations. Individually, the participants use the prototype and assess relevance of the obtained results in relation to their perceptions of the information need and the underlying simulated work task situation.

Table 1. Five tasks in four scenarios

\begin{tabular}{|l|c|}
\hline \multicolumn{1}{|c|}{ Tasks } & \multicolumn{1}{c|}{ Scenarios } \\
\cline { 1 - 1 } T1: Look for a particular exercise. & \multirow{2}{*}{ specific fact-finding } \\
\hline T2: Look for the e-mail of a course monitor. & \multirow{2}{*}{ extended fact-finding } \\
\hline $\begin{array}{l}\text { T3: Look for evaluation criteria recently } \\
\text { published by other professors. }\end{array}$ & open-ended browsing \\
\hline $\begin{array}{l}\text { T4: Look for a new approach used to teaching a } \\
\text { particular topic. }\end{array}$ & exploration of availability \\
\hline $\begin{array}{l}\text { T5: Look for the learning material available to a } \\
\text { particular course. }\end{array}$ & \\
\hline
\end{tabular}

The evaluation was comprised of four sessions as follows: (a) a survey session, (b) a training session, (c) a task session, and (d) an interview session. The survey was designed to collect demographic information and participant's experience with search tools and experience with CoTeia. A training session was given, including an overview of the prototype so that the participant could become familiar with the tools and the environment. After the training session, participants completed tasks while thinking aloud about their strategies. The participants were instructed to articulate what they are thinking and what they feel while working with the prototype. The utterances were registered using audio recording. In connection with each task, the interactions of the participants with the prototype were registered using log-file recording. All participants were requested to complete the same tasks. No time limit was set for any of the tasks. After each task participants answered questions about relevance of the results obtained and the usefulness of the prototype concerning that task. After performing the tasks, participants were interviewed for their perceptions of the prototype that they had experienced.

\subsection{Results and Observations}

After each task, participants completed a short questionnaire about the results obtained and the usefulness of the prototype for the task. The judgments were made 
on 7-point Likert scales $(1=$ none, $7=$ extreme $)$. The participants assigned an average rating of $5.92(\mathrm{SD}=0.90)$ for relevance and $6.07(\mathrm{SD}=0.81)$ for usefulness. These results support our first goal: the users could get relevant information using the prototype.

By analyzing the user log we found out that participants used different tools to complete the tasks. The average number of different tools per task was 3.24 (SD = 1.48). We have also observed different number of times tools were used, different choices of tools, and different sequences chosen to perform each task. Additionally, these differences were detected among participants for a same task. In general, the results confirmed our hypothesis for the second goal: users use different strategies. We intend to investigate the users' strategies in detail to improve the integration of each tool within the environment.

Fig. 2 shows how often the tools were actually used. Facet Browse and Keyword Search were the most used tools. The tools provided ways of search/retrieval results, and participants chosen to begin with Facet Browse $(62.5 \%)$ more frequently than with Keyword Search (37.5\%). Unexpectedly, Work Memory has a somewhat high percentage of usage; however, few participants (3) made extensive use of Work Memory while others (9) did not consider this tool in their tasks.

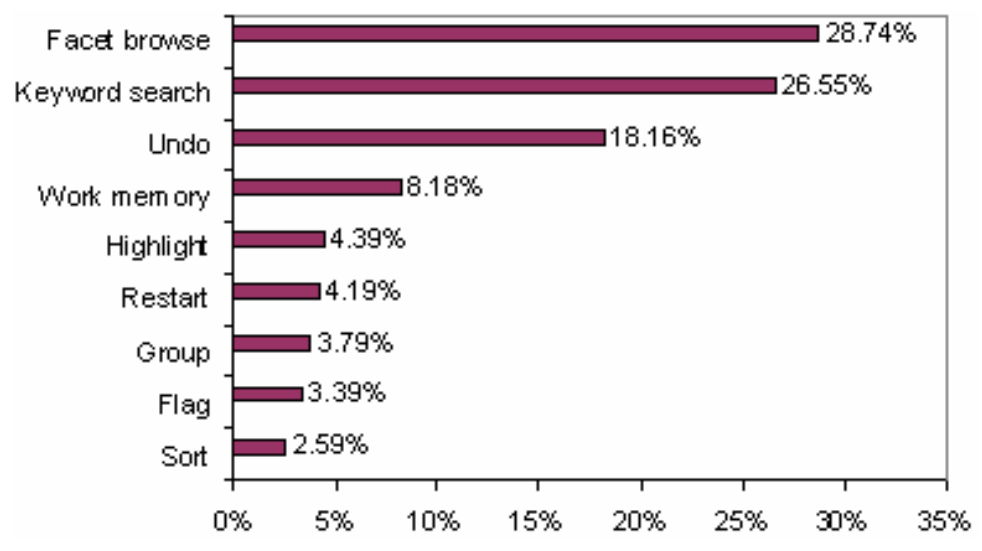

Fig. 2. Percentage of time tools were used

In general, participants considered available facets as representative of the wiki pages. Course (31.55\%), Learning Material Type (16.67\%), and Learning Activity Type $(11.90 \%)$ were the most used metadata in faceted browsing. One of the participants requested that the term (year/semester) concerning Course should be included in the interface. These results have motivated the need for specific vocabularies.

Participants commented favorably about the approach of the prototype and the available tools. Some (6) followed a strategy and after having completed the task they said that could improve the strategy. Participants were specifically asked to comment about the control of the navigation process. All participants stated that they felt in control by using the history feature. Furthermore, when asked participants did not 
stated getting lost while using the prototype. Initially, some participants (3) did not understand that, after cancel (undo) an action, the results reflect the resultant sequence of actions. However, as the participants continued to use the prototype, they perceived this feature and tried to exploit it. At the end of the interview, most of participants indicated they intend to use the prototype in the future.

\section{Related Work}

Many Semantic Wikis have been described in the literature, e.g. Semantic MediaWiki [4], Platypus Wiki [13], WikSAR [14], SemperWiki [15]. These Wikis use semantic information (metadata) to offer improved navigation, browsing, and searching. In general, they are primarily focused on semantic navigation (e.g. providing additional information on the relation a link describes) and semantic search (e.g. allowing a semantic query on the underlying knowledge base). SemperWiki has also provided faceted browsing [16]; however, it does not integrate this technique with others.

Our proposal integrates several tools in an environment to help browsing and searching. The prototype provides an interactive support to information seeking strategies based on orienteering. Users may choose an appropriate tool or a combination of tools to take steps along the way to satisfy their information needs.

\section{Conclusions}

Browsing for information in Wikis can become difficult as the number of pages and corresponding contents increases. We present a prototype developed to demonstrate our initial ideas combining searching tools to compose a strategy of orienteering. An evaluation showed that the prototype is useful to find relevant information in CoTeia repositories and provided insight regarding the tools and the underlying orienteering strategy.

Concerning future work, our main efforts address experiments with other types of user, in particular students, to investigate their interactions and performance using the prototype. We also plan to recognize some repeating strategies that can yield to patterns.

Acknowledgments. Our thanks to FINEP, FAPESP and UTFPR for funding parts of this research.

\section{References}

1. Teevan, J., Alvarado, C., Ackerman, M. S., Karger, D. R.: The perfect search engine is not enough: A study of orienteering behavior in directed search. In: Proc. Conference on Human Factors in Computing Systems (2004) 415-422

2. Arruda Jr, C. R. E., Izeki, C. A., Pimentel, M. G. C.: CoTeia: Uma ferramenta colaborativa de edição baseada na Web. In: Proc. 8th Brazilian Symposium on Multimedia and Hypermedia Systems (2002) 371-374 (in Portuguese) 
3. Guzdial, M.: Supporting learners as users. The Journal of Computer Documentation 23(2) (1999) 3-13

4. Völkel, M., Krötzsch, M., Vrandecic, D., Haller, H.: Semantic Wikipedia. In: Proc. 15th Int. Conf. on WWW (2006) 585-594

5. O'Day, V. L., Jeffries, R.: Orienteering in an Information Landscape: How Information Seekers Get From Here to There. In: Proc. ACM CHI (1993) 438-445

6. Bates, M. J.: Where should the person stop and the information search interface start? Information Processing and Management 26(5) (1990) 575-591

7. Navarro-Prieto, R., Scaife, M., Rogers, Y.: Cognitive strategies in Web searching. In: Proc. 5th Conference on Human Factors \& the Web (1999)

8. Hölscher, C., Strube, G.: Web search behavior of internet experts and newbies. In: Proc. 9th Conf. on WWW (2000)

9. Pansanato, L. T. E., Fortes, R. P. M.: Strategies for Filling Out LOM Metadata Fields in a Web-Based CSCL Tool. In: Proc. Third Latin American Web Congress (2005) 187-190

10. Pansanato, L. T. E., Fortes, R. P. M.: Strategies for automatic LOM metadata generating in a web-based CSCL tool. In: Proc. 11th Brazilian Symposium on Multimedia and the Web (2005) $1-8$

11. Shneiderman, B.: Designing information-abundant web sites: issues and recommendations. International Journal of Human-Computer Studies 47(1) (1997) 5-29

12. Borlund, P.: The IIR evaluation model: a framework for evaluation of interactive information retrieval systems. Information Research 8(3) (2003)

13. Tazzoli, R., Castagna, P., Campanini, S. E.: Towards a Semantic Wiki Wiki Web. In: Poster Track of the 3rd International Semantic Web Conference (2004)

14. Aumueller, D.: Semantic authoring and retrieval within a Wiki. In: Demos and Posters of the 2nd European Semantic Web Conference (2005)

15. Oren, E.: SemperWiki: a semantic personal Wiki. In: Proc. of the 1st Workshop on The Semantic Desktop, 4th International Semantic Web Conference (2005)

16. Oren, E., Delbru, R., Möller, K., Völkel, M., Handschuh, S.: Annotation and Navigation in Semantic Wikis. In: Proc. of the 1st Workshop on Semantic Wikis, 3rd European Semantic Web Conference (2006) 